\section{(る) OPEN ACCESS}

\title{
Short and long-term psychosocial consequences of participating in a colorectal cancer screening programme: a matched longitudinal study
}

\section{Jessica Malmqvist $\odot,{ }^{1,2}$ Volkert Dirk Siersma, ${ }^{1}$ Mie Sara Hestbech $\odot,{ }^{1}$ Christine Winther Bang, ${ }^{1}$ Dagný Rós Nicolaisdóttir, ${ }^{1}$ John Brodersen ${ }^{1,2}$}

\subsection{6/bmjebm-2020-111576}

- Additional online supplemental material is published online only. To view, please visit the journal online (http://dx.doi.org/ 10.1136/bmjebm-2020111576).

\section{${ }^{1}$ The Research Unit for General Practice and Section of General Practice, Department of Public Health, University of Copenhagen, Kobenhavn, Denmark ${ }^{2}$ Primary Health Care Research Unit, Region Zealand, Denmark}

\section{Correspondence to:} Dr Jessica Malmqvist, The Research Unit for General Practice and Section of General Practice, Department of Public Health, University of Copenhagen, Kobenhavn 1165, Denmark; jessica. malmqvist@sund.ku.dk

\section{Check for updates}

(1) Author(s) (or their employer(s)) 2021. Re-use permitted under CC BY-NC. No commercial re-use. See rights and permissions. Published by BMJ.

To cite: Malmqvist J, Siersma VD, Hestbech MS, et al. BMJ Evidence-Based Medicine Epub ahead of print: [please include Day Month Year]. doi:10.1136 bmjebm-2020-111576

\section{Abstract}

Objectives To investigate the psychosocial consequences of receiving a false-positive (no abnormalities) result or being diagnosed with polyps compared with receiving a negative result in a colorectal cancer (CRC) screening programme.

Design and setting This was a longitudinal study nested in the roll-out of the Danish CRC screening programme that targets all individuals aged 50-74 years.

Participants In the inclusion period (AprilSeptember 2017), all positive screenees $(n=1854)$ were consecutively enrolled and matched 2:1:1 on sex, age ( \pm 2 years), municipality and screening date with negative screenees $(n=933)$ and individuals not yet invited to screening $(n=933)$. Questionnaires were sent by mail to all eligible participants in Region Zealand, Denmark, after the screening result, 2 months and 12 months after the final result.

Positive screenees who did not receive the followup procedure were excluded.

Main outcome measures The primary outcomes were psychosocial consequences. Outcomes were measured with the CRC screening-specific questionnaire Consequences of screening in CRC with 11 outcomes after the screening result and with 21 outcomes at the two later assessments. Results After receiving the screening result, individuals with no abnormalities, low-risk and medium-risk and high-risk polyps scored significantly worse on 8 of 11 outcomes compared with the negative screenee group. At the 12-month follow-up, the differences were still significant in 8 of 21 outcomes (no abnormalities), 4 of 21 outcomes (low-risk polyps) and 10 of 21 outcomes (medium-risk and highrisk polyps). The negative screenee group and the group not yet invited to screening differed psychosocially on 5 of 11 outcomes after the screening result, but on none of the 21 outcomes at the 2 months and 12 months follow-up. Conclusions The study showed that there are both short-term and long-term psychosocial consequences associated with receiving a no abnormalities result or being diagnosed with polyps. The consequences were worst for individuals diagnosed with medium-risk and highrisk polyps.

\section{Summary box}

What is already known about this subject?

- Colorectal cancer (CRC) screening includes no abnormalities and findings of polyps.

- There are psychosocial consequences associated with receiving a cancer diagnosis through screening.

- Less is known about the psychosocial consequences of receiving a no abnormalities result or a diagnosis of polyps.

What are the new findings?

> This study showed that there were psychosocial consequences associated with receiving a result of no abnormalities or a diagnosis of polyps compared with a negative result and these consequences were present even a year after having received the result.

- An exposure-response relationship was identified, where individuals with medium-risk and high-risk polyps experienced more psychosocial consequences than no abnormalities and individuals with low-risk polyps.

How might it impact clinical practice in the foreseeable future?

- The results may be included in the information leaflet given people about CRC screening.

- The definition of polyps and the implications hereof may be more clearly communicated to the participants, to avoid unnecessary worry and anxiety.

- The results might be included in future evaluations of benefits and harms of CRC screening.

\section{Background}

Colorectal cancer (CRC) screening has been implemented in many countries in the last decade, including Denmark. ${ }^{1}$ There are different CRC 
screening procedures, and in Denmark and most other European countries a faecal immunochemical test (FIT) is used as the screening test and colonoscopy is the follow-up procedure. ${ }^{2}$ The colonoscopy has two purposes: to detect CRC at an early stage, and to detect colorectal polyps that most CRCs develop from. ${ }^{3-5}$ Although there is a clear association between CRC and polyps, most polyps never become malignant. ${ }^{36}$ In cases where the colonoscopy indicates the presence of polyps, polypectomy is performed. These participants are subsequently enrolled in postpolypectomy surveillance programmes according to the classification of their polyps. ${ }^{78}$

The procedures, the findings and diagnosis, and the treatment, prognosis and surveillance may have various levels of psychosocial consequences for the participant. ${ }^{9}$ Other cancer screening programmes have shown that receiving a cancer diagnosis and initiating therapy may have large consequences, and that a negative result may have a reassuring effect. ${ }^{10}{ }^{11}$ However, in CRC screening, less is known about the consequences of receiving a false-positive (no abnormalities) result or a diagnosis of polyps. ${ }^{12}$

A systematic review has previously investigated the psychosocial consequences of receiving a no abnormalities result in CRC screening. ${ }^{13}$ The narrative synthesis of the seven included studies found moderate, transient psychosocial consequences shortly before and after the follow-up colonoscopy. ${ }^{14-20}$ However, these studies used generic measures that may not have captured the changes in screening-specific psychosocial consequences that are relevant to the target population. ${ }^{21-23}$ Moreover, the results are difficult to interpret, since only one study used both a reference group and a no abnormalities result group. The other six studies either measured psychosocial consequences in a nondifferentiated positive screenee group (including true positives, no abnormalities and individuals with polyps) or they did not include a reference group.

Therefore, the objective of this study was to investigate the psychosocial consequences of receiving a no abnormalities result or a diagnosis of polyps compared with receiving a negative result in CRC screening with a questionnaire specifically developed and validated to measure the psychosocial consequences of CRC screening.

\section{Overall design and setting}

This study was a longitudinal survey nested in the roll-out of the Danish CRC screening programme and was conducted in Region Zealand, Denmark in the period of April 2017-February 2019 (figure 1).

The study groups were individuals with no abnormalities results, low-risk (LR) polyps and medium-risk and high-risk (MctHR) polyps. The reference groups were individuals with a true positive result and a negative result. These two groups are the groups expected to experience the most and the least psychosocial consequences, why these groups were used as benchmark groups.

Psychosocial consequences were the primary outcomes and were measured at three time points: shortly after the screening result, 2 months after the final result and 12 months after the final result (figure 2). The instrument used to measure psychosocial consequences was the consequences of screening for CRC questionnaire (COS-CRC). ${ }^{24}$

\section{The screening programme}

A population-based CRC screening programme was implemented in Denmark in 2014-2017, and targets all individuals aged 50-74 years with an FIT as the screening test (figure 1). ${ }^{8}$ The programme was rolled out gradually in a method based on birth months: the months of the year were randomly distributed over the 4 years, and each year individuals from three birth months were invited to participate in the screening.

Invitations to screening were sent by mail to eligible individuals each week. These invitations included a FIT-kit, a postagepaid return envelope, a CRC screening information brochure, and a reference to an opt-out webpage. Participants performed the test at home and sent the test sample by mail to the regional screening unit laboratory for analysis. Positive screenees received a letter with the test result, a prebooked colonoscopy (within 14 days) that could be rescheduled by the participant, and a bowel cleansing kit with instructions for bowel preparation. Bowel preparation is done at home the day prior to colonoscopy. During the colonoscopy, participants are offered sedatives if needed.

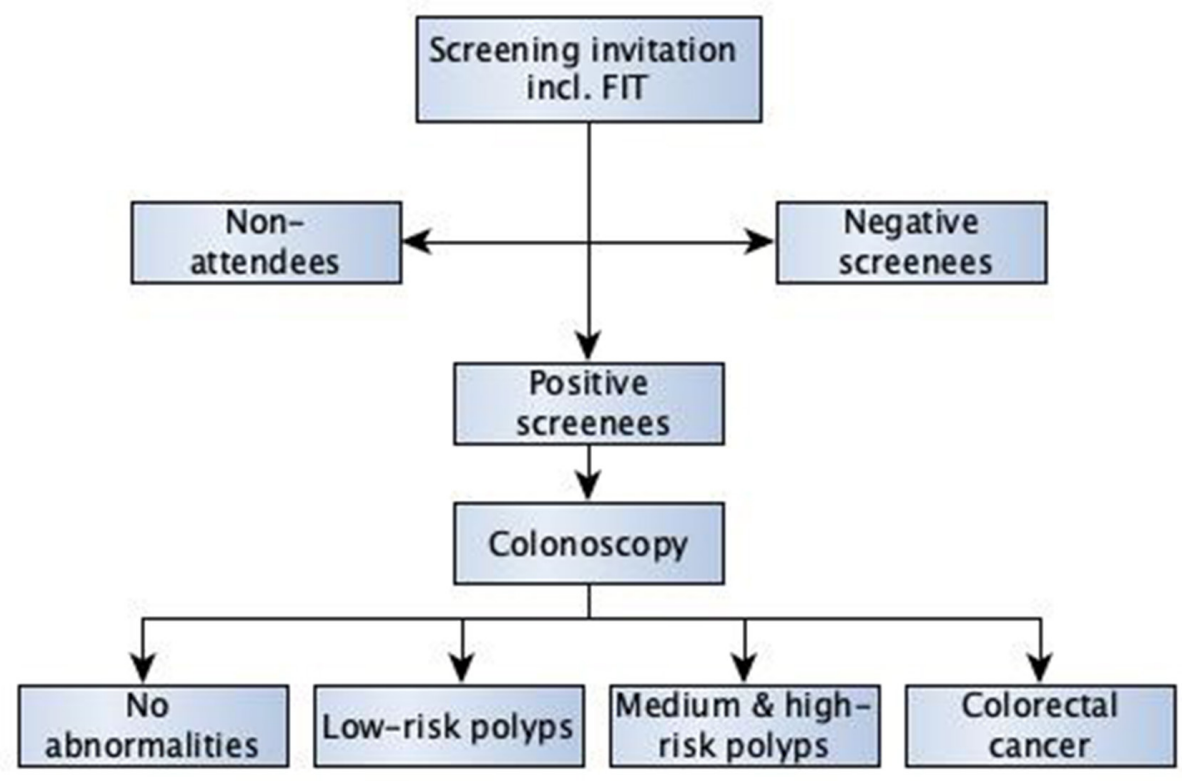

Figure 1 The Danish CRC screening programme. CRC, colorectal cancer FIT, faecal immunochemical test. 


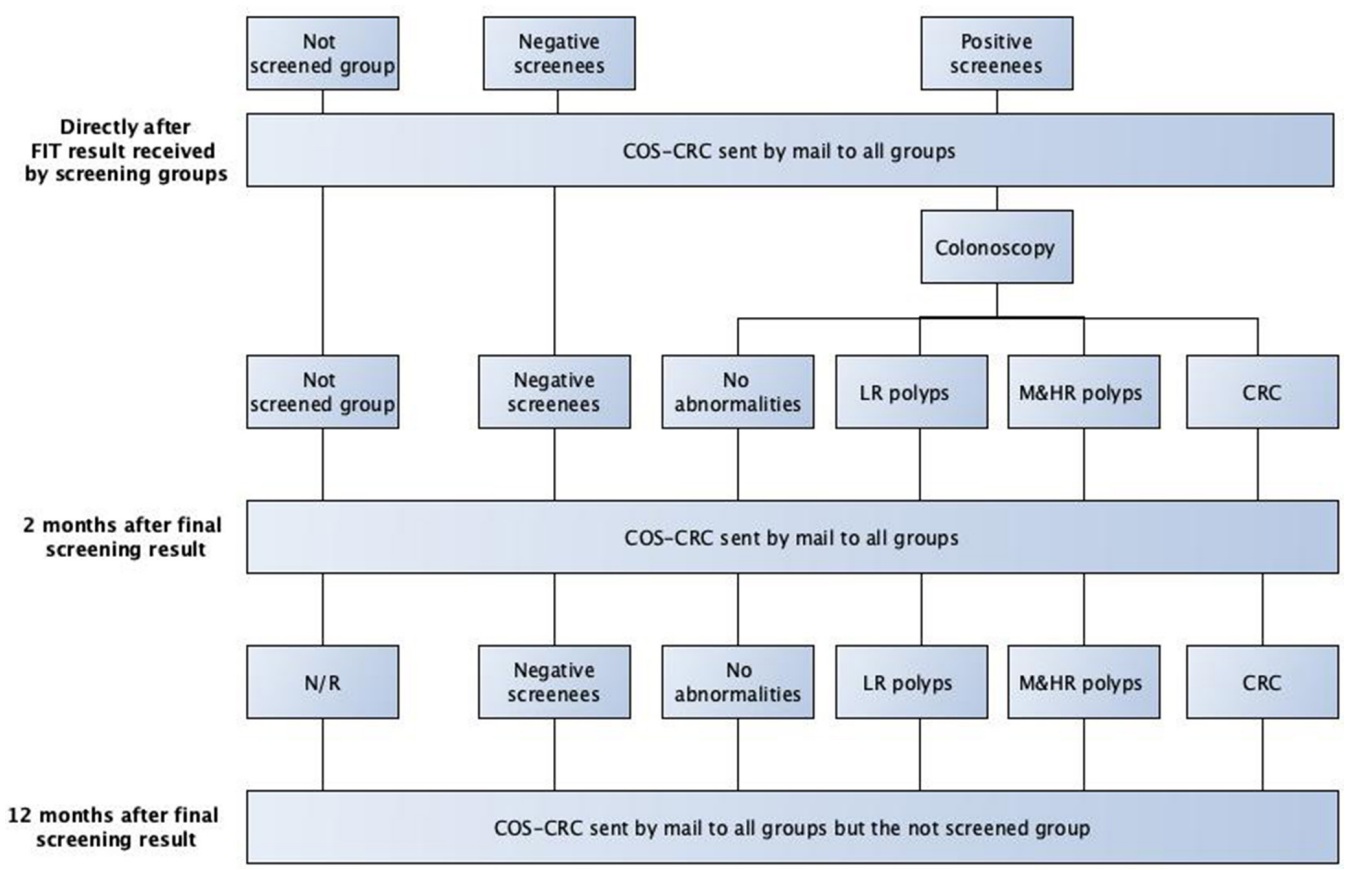

Figure 2 Inclusion diagram. COS-CRC, consequences of screening-colorectal cancer; FIT, faecal immunochemical test; LR, low risk; N/R, not reported.

The colonoscopy results in the following diagnoses: no abnormalities, LR polyps, medium-risk and high-risk (MEtHR) polyps and CRC (true-positive result). Individuals with no abnormalities receive their result on site, while in cases of polyp findings or cancer-suspicious lesions, biopsies are performed and results are received after pathological investigation. Individuals with M\&tHR polyps are referred to a polyp surveillance programme with follow-up colonoscopies 1-3 years later. Individuals with CRC are referred to a fast-track cancer pathway for further investigations and treatment. ${ }^{25}$

On 31 December 2017, the screening programme was completely rolled out and the target population is now invited to screening biennially.

\section{Study population}

The psychosocial consequences were compared between three main cohorts: positive screenees, subdivided into no abnormalities, LR polyps, MEtHR polyps and CRC; negative screenees and the not screened group (figure 2). The definition of the polyp categories were:

- LR polyps: less than three in number and less than $10 \mathrm{~mm}$ in size; tubular adenomas or low-grade neoplasia.

- MR polyps: one adenoma $\geq 10 \mathrm{~mm}$ but $<20 \mathrm{~mm}$ in size, three to four adenomas regardless of size, tubulovillous or villous adenomas; high-grade neoplasia.

- HR polyps: one adenoma $\geq 20 \mathrm{~mm}$ in size, five or more polyps regardless of size, or one polyp resected with piecemeal technique (too large to resect in one piece).

Tentatively, the negative screenees and the true-positive (CRC) group were used as benchmark groups, expected to experience the least and the worst consequences, respectively.

The choice of subgroups was based on the different treatments, surveillance programmes, and screening options these groups were offered. ${ }^{8}$ Moreover, we took into account what degree of psychosocial consequences the terminology of these diagnoses might have.

\section{Data collection}

From 28 April 2017 to 29 September 2017, we obtained contact information on all positive screenees consecutively twice a week from the regional screening unit. For each positive screenee, we also received contact information for a negative screenee, and a not yet screened individual matched on sex, birth year ( \pm 2 years), screening date (only negative screenees) and municipality. The screening cohort was invited to screening in the first 9 months and the not screened group in the last 3 months of 2017.

We sent the COS-CRC questionnaire, a postage-paid return envelope and an information letter about the purpose of the study by mail to the three cohorts within 2 weekdays after the positive screenees received their FIT results (figure 2). A reminder was sent 3 weeks later to all but the positive cohort, since most participants in the positive cohort had undergone the colonoscopy at that time.

Two months and 12 months after the positive screenees received their colonoscopy results, all the cohorts received the questionnaire again. In February 2019, data collection for the 12-month follow-up was finished. The participant's consent was obtained by responding to the questionnaire and withdrawal of consent was obtained by mail, email and phone.

The not screened group was invited to CRC screening between October and December 2017. Hence, this group had been screened at the 12-month follow-up and were not sent the COS-CRC at this time. Since the not screened group was scheduled to be screened in the middle of the 2-month follow-up (which began in October 2017), we stopped data collection in this group on 30 September 2017.

Therefore, the negative screenee group was used as a reference group at all three measurements. This choice was further justified since the negative screenee group was the group expected to experience the fewest negative psychosocial consequences.

At the date for invitation to screening, we obtained sociodemographic characteristics from the Danish electronic registers, Statistics Denmark. 
Since we aimed to achieve age-distributed comparable cohorts, people turning 50 or 75 in the year of invitation to screening were omitted from our analyses. Moreover, we excluded individuals who did not undergo the follow-up procedure after a positive FIT. The latter group included individuals who were already involved in CRC surveillance programmes, individuals who were not eligible for any follow-up procedure, or individuals who declined the offer of the follow-up colonoscopy. A qualitative study on experiences of screening in this specific group has been published elsewhere. ${ }^{26}$

Drop-out was defined as active withdrawal of consent, and non-response as a passive act of not responding to one or more questionnaires.

\section{Questionnaire}

We used the condition-specific questionnaire COS-CRC to assess the psychosocial consequences. ${ }^{24} \mathrm{COS}-\mathrm{CRC}$ is an extended version of the screening-specific questionnaire $\mathrm{COS}^{27}{ }^{28}$ COS-CRC was developed in two phases: (1) in the first phase, we performed focus groups with CRC screening participants with no abnormalities and with LR polyp findings to ensure high content validity; (2) in the second phase, data collected in this study was used to validate the questionnaire statistically using item response theory and Rasch Models. $^{29}$

COS-CRC consists of three parts: part I that consists of nine scales and two single items and can be used at any time point in the screening cascade and in a group not invited to screening as well, part Ix that consists of four scales and one single item and is only relevant to participants that have undergone the follow-up colonoscopy and part II that consists of five scales, and measures long-term consequences of CRC screening. Hence, it is relevant to all screening participants who have received a final screening result. In online supplemental materials 2 and 3, overviews of the different scales, number of items for each scale and the response categories are presented.

\section{Statistics and data management Questionnaire data}

Questionnaire data were typed twice by two independent administrative personnel. Subsequently, we linked data and analysed for differences in values for each item. Errors were identified and corrected by rereading the original questionnaires. If any item in a questionnaire was not completed, the scale to which the item belonged was defined as missing. We defined a questionnaire as responded to if one item was completed. All questionnaires defined as responded were included in the statistical analyses independent of whether the participant was later a non-respondent or dropped out of the study later.

\section{Covariates}

We performed unadjusted analyses and analyses adjusted for the following covariates: sex, age, urbanicity, educational level, annual income, wealth, employment status, cohabitation status (living alone yes/no) and Charlson's Comorbidity Index (CCI). CCI is a weighted index taking the number and seriousness of comorbid diseases into account. ${ }^{30}$ The higher the score the higher the risk of death from comorbid diseases.

Urbanicity was subdivided into three categories, (1) capital city: more than $50 \%$ of the residents in a municipality live in a densely populated area (more than 40000 residents in the largest city), (2) small town: less than 50\% of the residents in that municipality live in a densely populated area and less than 50\% of the residents live in sparsely populated areas (less than 15000 residents in the largest city) (3) rural area: more than 50\% of the residents in the municipality live in sparsely populated areas.

Educational level was categorised into: (1) elementary school (10 years); (2) secondary school including vocational education; (3) higher education-short (2 years at business academies) and higher education-medium (3-4.5 years at university colleges); (4) higher education-long (bachelors, masters, $\mathrm{PhD}$ at university) and (5) no education registered.

Annual income was defined as all taxable income except investment income categorised into: (1) €26 800 or less; (2) €26 800-€40 200; (3) €40 201-€67 000 and (4) above €67 000. ${ }^{31}$

The covariate 'wealth' was defined with the lowest category according to the governmental aid corresponding to a maximum of $€ 350$ given to retired persons with a wealth below $€ 67000$. The highest category was defined from the cut-off for the higher tax bracket on wealth and income in Denmark.

Employment status was defined as: employed (self-employed individuals and employees); unemployed (including students over 15 years and individuals receiving social transfers) and retired.

\section{Statistical analyses}

Analyses of baseline characteristics were performed with analysis of variance test (continuous variables) and $\chi^{2}$ test (categorical variables).

We performed the analyses of psychosocial consequences in multivariable linear regression models. Adjustment for repeated measures and weighting was done with generalised estimating equations methods. Besides adjusting for the previously mentioned covariates, we adjusted for different non-response types and rates between the cohorts by weighting observations that were available at each follow-up time point by the inverse of the probability of not being missing. ${ }^{32}$ The latter is estimated from logistic regression models including the above potential confounders and outcomes at previous time points. We performed the analyses in SAS V. 9.4 (SAS Institute) and R V. 3.5.0. We adjusted for multiple testing by using the Benjamini-Hochberg procedure. ${ }^{33}$

\section{Sample size}

Previous studies using COS have shown that the sensitivity of COS is high enough to measure differences between 500 individuals in the general population. ${ }^{10} 3435$

Moreover, the sensitivity of the questionnaire is sufficient to measure a difference between 100 participants receiving a no abnormalities result and 100 participants receiving a negative screening result. ${ }^{28}$

In the implementation period of the CRC screening programme, the distribution of colonoscopy findings was: no abnormalities 33.7\%, LR polyps 18.6\%, MAtHR polyps 32.0\%, CRC 5.9\%. In $9.8 \%$ the colonoscopy result had not been registered in the CRC screening database. ${ }^{36}$ This division into positive subcohorts according to the colonoscopy results requires a larger sample size of the positive group than of the negative group and the not screened group to achieve a final distribution of at least 100 respondents in each subcohort.

In breast cancer screening studies, the response rates have been approximately 90\% for positive screenees, and approximately 70\% for negative screenees and for individuals not invited to screening. We expected a lower response rate in our mixed population.

All these estimates were taken into account when defining the sample size. ${ }^{10} 112834$ According to the predefined smaller sample size estimate for negative screenees and the not screened group compared with the positive group, we performed another 2:1:1 


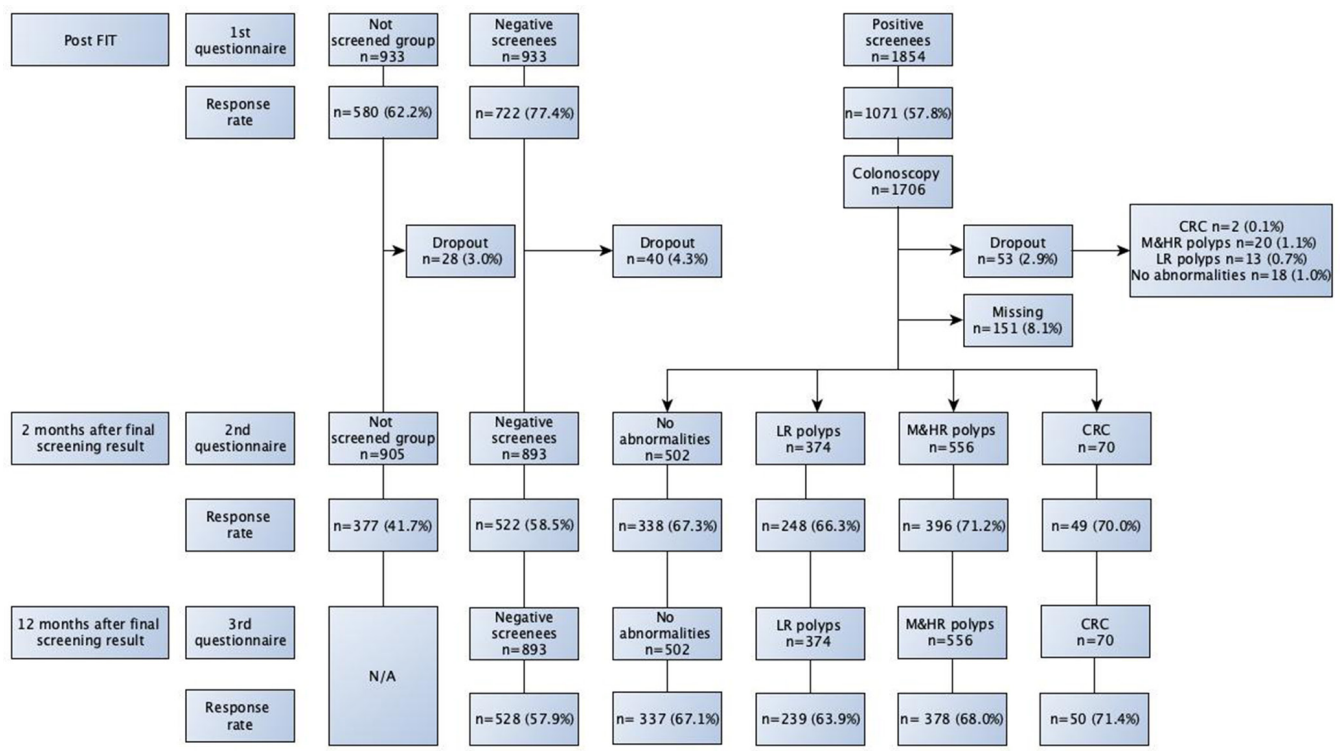

Figure 3 Response rates. CRC, colorectal cancer; FIT, faecal immunochemical test; LR, low risk; N/A, not available.

matching with the positive group, negative group, and the not screened group, thus sending the COS-CRC to approximately half of the negative screenees and the not screened group.

\section{Patient and public involvement}

Neither patients nor the public were involved in the design, or conduct, or reporting, or dissemination plans of our research. Screening participants were involved in the development of the questionnaire used in this study.

\section{Results}

The flow chart of the inclusion process and response rates are illustrated in figures 2 and 3. The initial response rates were high in the negative screenee group (77.4\%) and the not screened group $(62.2 \%)$ but thereafter a trend of decreasing response rates in the follow-up assessments was seen; 58.5\%, 57.9\% for negative screenees and $41.7 \%$ for the not screened group. In contrast, a trend of increasing response rates was seen in the cohorts with positive screening results over time; $57.8 \%$ at the first assessment and $63.9 \%-71.4 \%$ at the follow-up assessments.

Table 1 presents sociodemographics for the three main cohorts at the time of enrolment. The study groups differed significantly in the covariates age, educational level, wealth, CCI and living alone. There was a 1-year difference in mean age with individuals in the not screened group being younger than screenees. In the variables educational level, wealth, CCI, and living alone, negative screenees had a more favourable status than positive screenees and not screened individuals, meaning that negative screenees had a higher educational level, a larger wealth, lower CCI score and were to a lesser extent living alone than positive screenees and not screened individuals.

The tendencies seen in figure 4 are mimicked by the estimates of the mean differences adjusted for covariates and potential confounders in online supplemental material 1, table 2.

\section{After the screening result}

The no abnormalities group, the LR polyp group, and the MAtHR polyp group all had significantly worse (higher) scores than the negative screenee group in all scales but one, 'emotional reactions' (online supplemental material 1, table 2). The not screened group had significantly worse scores compared with the negative screenee group in three scales and the two single items.

\section{Two-month follow-up}

There was a tendency of decrease in negative psychosocial consequences from after the screening result to this assessment for all three groups: no abnormalities, LR polyp group and MAtHR polyp group (online supplemental material 1, table 2). The MAtHR polyp group was worst off, with significantly worse scores in seven part I scales, and one single part I item. All three groups had significantly different scores in three of five part II scales than negative screenees.

The MAHR polyp group was more uncertain than the LR polyp group of what implications a diagnosis of polyps had.

At this assessment, the not screened group and negative screenee group did not differ in psychosocial status.

\section{Twelve-month follow-up}

There was also a tendency of decrease in psychosocial consequences in the three groups: no abnormalities, LR polyps and MctHR polyps from the 2-month follow-up.

The abnormalities group and the MAtHR polyp group still experienced more negative psychosocial consequences in three and five part I scales, respectively. Both groups were still more dejected, anxious and had more sleep disturbances than negative screenees, while individuals in the MatHR polyp group also continued to be more introverted and felt more fear and lack of power than negative screenees.

All three groups experienced significantly more negative psychosocial consequences than the negative screenees in four (no abnormalities) and three (LR and MctHR polyps) of five part II scales. All three groups continued to experience changes in calmness, existential values and empathy, while the no abnormalities group also experienced changes in their social relations.

The MAtHR polyp group was still more uncertain about the implications of their polyp findings than the LR polyp group.

\section{Discussion}

The results of this study showed that there were statistically significantly more short-term and long-term psychosocial 


\begin{tabular}{|c|c|c|c|c|}
\hline & $\begin{array}{l}\text { Positive screening result } \\
n=1854(49.8 \%)\end{array}$ & $\begin{array}{l}\text { Negative screening result } n=933 \\
(25.1 \%)\end{array}$ & $\begin{array}{l}\text { Not screened group } n=933 \\
(25.1 \%)\end{array}$ & Pvalue* \\
\hline Sex, n (\%) & & & & 0.5860 \\
\hline Male & $1051(56.7)$ & $534(57.2)$ & $548(58.7)$ & \\
\hline Female & $803(43.3)$ & $399(42.8)$ & $384(41.3)$ & \\
\hline Age, mean (SD) & $65.5(5.9)$ & $65.5(5.9)$ & $64.5(5.9)$ & $<0.0001$ \\
\hline Urbanicity, n (\%) & & & & 0.8192 \\
\hline Capital city & $127(6.8)$ & $57(6.1)$ & $56(6.0)$ & \\
\hline Small town & $749(40.4)$ & $377(40.4)$ & $367(39.3)$ & \\
\hline Rural area & $978(52.8)$ & $499(53.5)$ & $510(54.7)$ & \\
\hline Educational level, n (\%) & & & & 0.0033 \\
\hline Elementary school & $531(28.6)$ & $221(23.7)$ & $268(28.7)$ & \\
\hline Secondary school & $900(48.6)$ & $435(46.6)$ & $425(45.6)$ & \\
\hline Short higher education & $325(17.5)$ & $205(22.0)$ & $185(19.8)$ & \\
\hline Long higher education & $67(3.6)$ & $57(6.1)$ & $41(4.4)$ & \\
\hline None registered & $31(1.7)$ & $15(1.6)$ & $14(1.5)$ & \\
\hline Employment status, n (\%) & & & & 0.0646 \\
\hline Employed & $644(34.7)$ & $345(37.0)$ & 354 (37.9) & \\
\hline Unemployed & $199(10.8)$ & $81(8.7)$ & $110(11.8)$ & \\
\hline Retired & $1011(54.5)$ & $507(54.3)$ & $469(50.3)$ & \\
\hline Annual income, $n$ (\%) & & & & 0.0935 \\
\hline$\langle € 26800$ & $912(49.2)$ & $408(43.7)$ & $445(47.7)$ & \\
\hline$€ 26800-40200$ & $514(27.7)$ & $263(28.2)$ & $252(27.0)$ & \\
\hline$€ 40201-67000$ & $343(18.5)$ & $205(22.0)$ & $187(20.0)$ & \\
\hline ১€67000 & $85(4.6)$ & $57(6.1)$ & $49(5.3)$ & \\
\hline Wealth, n (\%) & & & & $<0.0001$ \\
\hline$\left\langle € 11800^{\star}\right.$ & $722(38.9)$ & $301(32.3)$ & $392(42.0)$ & \\
\hline$€ 11800-67000$ & $415(22.4)$ & $195(20.9)$ & $196(21.0)$ & \\
\hline 〉€67000 & $717(38.7)$ & $437(46.8)$ & $345(37.0)$ & \\
\hline CCl, mean (SD) & $0.7(1.4)$ & $0.5(1.1)$ & $0.6(1.3)$ & 0.0004 \\
\hline Living alone, $\mathrm{n}(\%)$ & & & & $<0.0001$ \\
\hline No & $1333(71.9)$ & $741(79.4)$ & $670(71.8)$ & \\
\hline Yes & $521(28.1)$ & $192(20.6)$ & $263(28.2)$ & \\
\hline
\end{tabular}

*P value of a $\chi^{2}$ test (categorical variables) or an ANOVA test (continuous variables); the Benjamini-Hochberg procedure rejects all $\mathrm{p}$ values above 0.023 to control the false discovery rate at 0.05 .

ANOVA, analysis of variance; CCl, Charlson's Comorbidity Index.

consequences associated with receiving a no abnormalities result or being diagnosed with polyps than receiving a negative result in a CRC screening programme. Moreover, an exposure-response relationship was identified; individuals diagnosed with MEtHR polyps experienced more negative psychosocial consequences than individuals with no abnormalities, who experienced more negative consequences than individuals with LR polyps.

Sociodemographically, the negative group was significantly better off than the not screened group and the positive screenees. This could have exaggerated the psychosocial differences between the negative screenee group and the positive subcohorts. However, since the not screened group and negative screenee group did not differ significantly in psychosocial consequences at the 2-month follow-up, sociodemographics do not seem to have affected the results.

At the first assessment, there were differences between the not screened group and the negative screenees in the scales 'Behaviour', 'Sleep' and the two single items 'Lifestyle changes' and 'Impact on sexuality. A plausible explanation for this is the suggested reassurance negative screenees may have experienced when receiving a negative screening result. ${ }^{10}$
The no abnormalities group scored worse on more scales than the LR polyp group both at 2-month follow-up and at 12-month follow-up. This could be explained by the fact that receiving a diagnosis of LR polyps gives the participant a plausible and harmless explanation for the positive screening result, while a no abnormalities result could leave the participant with a feeling of uncertainty about what caused the initial positive result and a query about whether the colonoscopist might have missed something. ${ }^{37}$

There was a trend of decrease in psychosocial consequences with time in all the positive subgroups, which was expected. However, even a year after the final screening result the MctHR polyp group scored significantly different on five part I scales, and three part II scales than the negative screenee group. More interestingly, the group with no abnormalities scored significantly different than the negative screenee group on three part I scales, one part I single item 'Impact on sexuality' and four part II scales.

To better understand the meaning of the results, the scale 'Anxiety' (including seven items, range of values $0-21$ ) can be used as an example. The no abnormalities group scored 0.58 points higher (worse) compared with the negative screenee group on this 

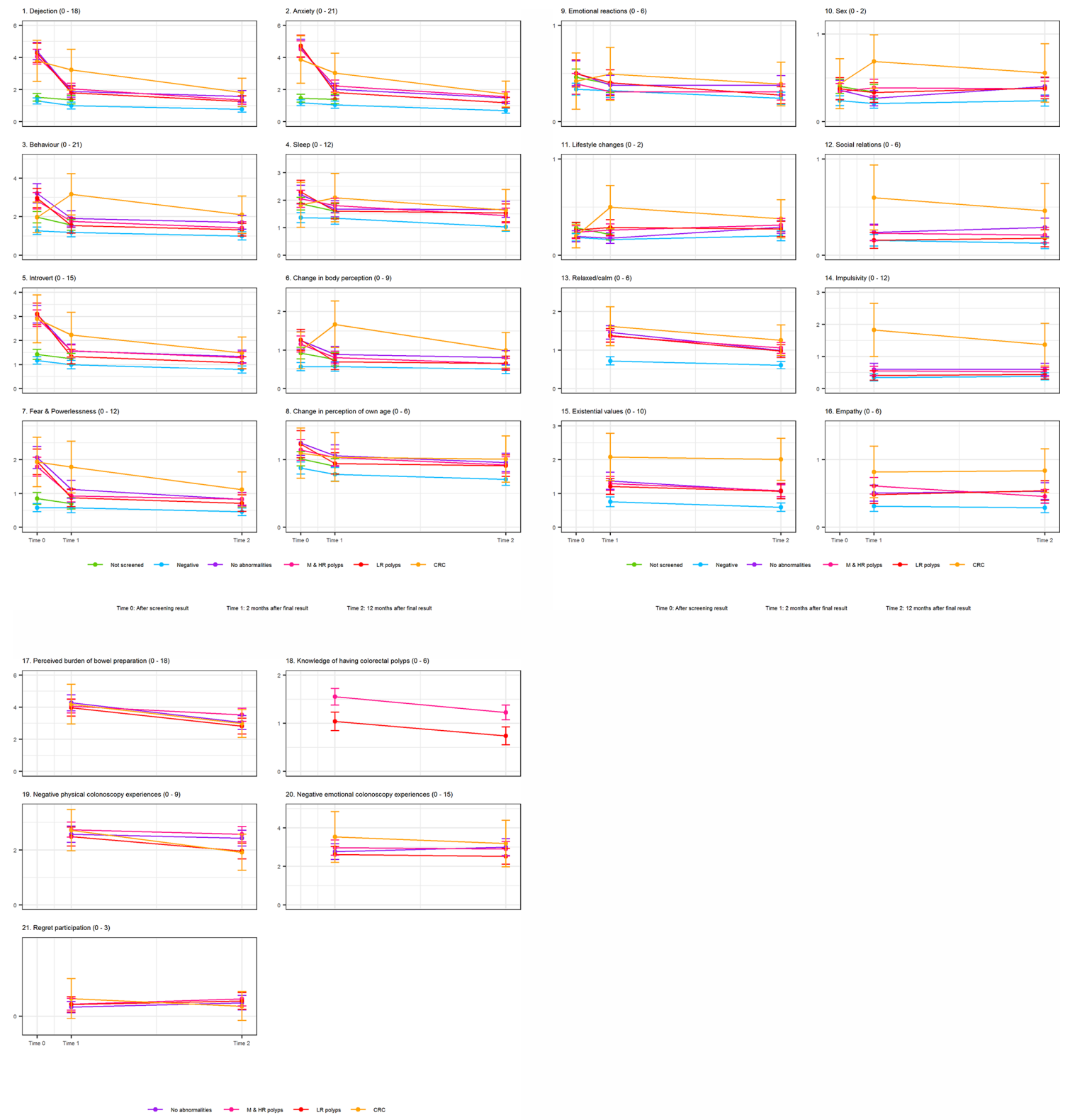

Figure 4 Mean COS-CRC scale scores for the study groups and benchmark groups at each of the time points

scale 12 months after having received their final screening result (online supplemental material 1, table 2). This could be achieved by $58 \%$ of participants in the no abnormalities group scored 'a bit' on one of seven items while the negative result group scored 'not at all' on all seven items, a description of the effect size that may be better interpreted.

It is uncertain what the aforementioned subtle changes in psychosocial status have of importance and thereby impact on the individual and society. ${ }^{23} 38$ However, we have used the negative group and the true-positive group as our two benchmarks and revealed robust evidence that having a positive CRC screening results leading to the final diagnoses of no abnormalities, LR polyps and MCtHR polyps have long-term negative psychosocial consequences 1 year after these final diagnoses. Future research has to investigate if these psychosocial changes relate to other harms, for example, overutilisation of healthcare and thereby waste of resources and additional unintended derived harms. ${ }^{23} 3940$
Since the importance of the results are uncertain, it is difficult to compare them with the screening benefits reported in a recent update of a Cochrane review which showed that biennial screening with guaiac fecal occult blood test (no data for FIT were available) reduced CRC cases by one (three fewer to one more) and CRC deaths by one (two fewer to one fewer) in a 15-year perspective compared with no screening. ${ }^{41}$

The longitudinal design with a reference group and positive subcohorts, and timely, concurrent assessments are considerable strengths of this study. Use of a condition-specific questionnaire with high content validity and adequate measurement properties is also an essential strength. Moreover, we had a high overall response rate in the three study cohorts: no abnormalities, LR polyps and MetHR polyps.

This study also had several limitations. The rate of CRC was $3.8 \%$ in our data, but we determined our sample size from a CRC rate of $6 \% .{ }^{36}$ Since the CRC group was much smaller than expected, 
the estimate for psychosocial consequences in this group is associated with large uncertainty and is ill-determined. Another limitation is the contamination of our not screened group. The use of a control group instead of a reference group (ie, negative screenees who had received the reassurance of being healthy) may have diminished the differences. However, since there were no differences between the not screened group and the negative screenee group at the 2-month follow-up we are confident that the overall results would not have changed with a control group. ${ }^{10}$

We did not perform a baseline assessment before screening for economic and pragmatic reasons, which can also be regarded as a limitation. To reach an effect size of minimum 100 positive screenees in each subcohort (no abnormalities, LR polyps, MAtHR polyps, CRC) estimated from previous screening studies using COS, we should have enrolled approximately 40000 invitees. $^{22}{ }^{23}$ However, we have performed a pseudobaseline assessment by performing an invitation study prior to this study in another sample of the screening target population (paper accepted for publication, awaiting DOI). This study did not show any association between being invited to CRC screening and experiencing negative psychosocial consequences. These results also support the use of the negative screenees as our reference group.

Furthermore, we did not calculate a minimal important difference (MID) prior to this study. ${ }^{42}$ Calculation of MID requires an assumption of differences being the same across the scale of response categories. Hence, a change in anxiety should mean the same for a person diagnosed with CRC as for a person with a no abnormalities result. Moreover, calculation of an MID would require that a change in units on every scale of COS-CRC means the same, for example, a change from 'a bit' to 'not at all' would mean the same in all scales in COS part I and part Ix. Therefore, we used benchmark groups (negative result group and CRC group) which tentatively were the least and most psychosocially affected.

Our results in the no abnormalities group are different from previous research since this subgroup experienced negative psychosocial consequences even a year after the colonoscopy, while previous studies have not shown longer-term consequences. ${ }^{13}$ Since other studies have used generic rather than condition-specific questionnaires, they might not have captured the subtle changes in psychosocial consequences which could be an explanation for the identified differences. ${ }^{23}$

The MAtHR polyp group experienced more negative psychosocial consequences than the no abnormalities group and the LR polyp group at both the 2-month and the 12-month follow-up. Only few previous studies have investigated psychosocial outcomes after having received a polyp diagnosis. ${ }^{144}$ One study did not find any differences in anxiety between the adenoma group and CRC group. The objective of our study was not to compare psychosocial consequences between these two groups and therefore we cannot rule out that this is applicable to our study as well. The other study found an increased cancer-specific worry in individuals with no abnormalities and LR polyps. However, since the study design, outcome measures and comparative groups differed it is difficult to interpret and compare the results. This is a general challenge in studies measuring psychosocial consequences. Although research has suggested use of a longitudinal design, timely assessments in all subgroups and use of condition-specific instruments to measure the psychosocial consequences, few studies have adapted this strategy. ${ }^{132223}$

The results of this study add important knowledge to the field of harms of CRC screening, and especially to the gap in knowledge about the psychosocial consequences of receiving a diagnosis of colorectal polyps.
Since all harm domains of CRC screening have not yet been investigated, it is unclear what public health importance the results of this study may have. ${ }^{3845}$

Furthermore, as the magnitude of the psychosocial consequences may depend on the screening context, the generalisability to other general population CRC screening settings using FIT as the screening method are still of uncertainty.

\section{Conclusion}

This study showed that statistically significantly more short and long-term psychosocial consequences were associated with receiving a no abnormalities result or being diagnosed with polyps. Moreover, an exposure-response relationship was identified; individuals diagnosed with CRC experienced more negative psychosocial consequences than individuals with M\&tHR polyps, who experienced more negative psychosocial consequences than individuals with individuals with no abnormalities, who experienced more negative consequences than individuals with LR polyps. What these subtle changes have of importance, and how to compare them with the intended screening benefits are to be researched.

Acknowledgements We want to thank all the participants for their contribution to this research. We also want to thank all the working students who have helped us in different phases of the project.

Contributors JM, JB and VDS were involved in the study design and concept of the study. JM was responsible for the data collection and typing. DRN was responsible for cleaning of data and preparing the data sets. VDS and CWB performed the statistical analyses. JM interpreted the results and drafted the manuscript. All authors contributed with critical revisions and important scientific content of the manuscript. JM and JB are guarantors of the study. The corresponding author confirms that all listed authors fulfil authorship criteria and that no others fulfilling the criteria have been omitted.

Funding This project was funded by the Region Zealand Foundation grant numbers 15-000342 and 13-000849.

Disclaimer The funding source had no role in study design, data collection and analysis, decision to publish, or preparation of the manuscript.

Competing interests None declared.

Patient consent for publication Not required.

Ethics approval The study has been registered by the Danish Data Protection Agency 12 January 2016 (file no. 2015-41-4514 and 2014-54-0804) and by the Danish Patient Safety Authority 13 September 2016 (file no.: 3-3013-1753/1/).

Provenance and peer review Not commissioned; externally peer reviewed.

Data availability statement Data are available on reasonable request. Data and questionnaires are available on reasonable request from the corresponding author.

Supplemental material This content has been supplied by the author(s). It has not been vetted by BMJ Publishing Group Limited (BMJ) and may not have been peer-reviewed. Any opinions or recommendations discussed are solely those of the author(s) and are not endorsed by BMJ. BMJ disclaims all liability and responsibility arising from any reliance placed on the content. Where the content includes any translated 
material, BMJ does not warrant the accuracy and reliability of the translations (including but not limited to local regulations, clinical guidelines, terminology, drug names and drug dosages), and is not responsible for any error and/or omissions arising from translation and adaptation or otherwise.

Open access This is an open access article distributed in accordance with the Creative Commons Attribution Non Commercial (CC BY-NC 4.0) license, which permits others to distribute, remix, adapt, build upon this work non-commercially, and license their derivative works on different terms, provided the original work is properly cited, appropriate credit is given, any changes made indicated, and the use is non-commercial. See: http://creativecommons.org/licenses/by-nc/4.0/.

\section{ORCID iDs}

Jessica Malmqvist http://orcid.org/0000-0002-9299-4369

Mie Sara Hestbech http://orcid.org/0000-0001-8247-3642

\section{References}

1 Benson VS, Atkin WS, Green J, et al. Toward standardizing and reporting colorectal cancer screening indicators on an international level: the International colorectal cancer screening network. Int J Cancer 2012;130:2961-73. doi:10.1002/ijc.26310

2 Navarro M, Nicolas A, Ferrandez A, et al. Colorectal cancer population screening programs worldwide in 2016: an update. World J Gastroenterol 2017;23:3632-42. doi:10.3748/wjg.v23.i20.3632

3 Øines M, Helsingen LM, Bretthauer M, et al. Epidemiology and risk factors of colorectal polyps. Best Pract Res Clin Gastroenterol 2017;31:419-24. doi:10.1016/j.bpg.2017.06.004

4 Colucci PM, Yale SH, Rall CJ. Colorectal polyps. Clin Med Res 2003;1:261-2. doi:10.3121/cmr.1.3.261

5 Pickhardt PJ. The natural history of colorectal polyps and masses: rediscovered truths from the barium enema era. AJR Am J Roentgenol 2007;188:619-21. doi:10.2214/AJR.06.0731

6 Vleugels JLA, Hazewinkel Y, Fockens P, et al. Natural history of diminutive and small colorectal polyps: a systematic literature review. Gastrointest Endosc 2017;85:1169-76. doi:10.1016/j.gie.2016.12.014

7 Atkin WS, Valori R, Kuipers EJ, et al. European guidelines for quality assurance in colorectal cancer screening and diagnosis. First Edition-Colonoscopic surveillance following adenoma removal. Endoscopy 2012;44 Suppl 3:SE151-63.

8 Njor SH, Friis-Hansen L, Andersen B, et al. Three years of colorectal cancer screening in Denmark. Cancer Epidemiol 2018;57:39-44. doi:10.1016/j. canep.2018.09.003

9 Cotter AR, Vuong K, Mustelin L, et al. Do psychological harms result from being labelled with an unexpected diagnosis of abdominal aortic aneurysm or prostate cancer through screening? A systematic review. BMJ Open 2017;7:e017565.

10 Brodersen J, Siersma V, Ryle M. Breast cancer screening: "reassuring" the worried well? Scand J Public Health 2011;39:326-32. doi:10.1177/1403494810396558

11 Brodersen J, Siersma VD. Long-Term psychosocial consequences of falsepositive screening mammography. Ann Fam Med 2013;11:106-15.

12 Hagger MS, Orbell S. Illness representations and emotion in people with abnormal screening results. Psychol Health 2006;21:183-209.

13 van der Velde JL, Blanker MH, Stegmann ME, et al. A systematic review of the psychological impact of false-positive colorectal cancer screening: what is the role of the general practitioner? Eur J Cancer Care 2017;26. doi:10.1111/ecc.12709. [Epub ahead of print: 1105 2017].

14 Orbell S, O'Sullivan I, Parker R, et al. Illness representations and coping following an abnormal colorectal cancer screening result. Soc Sci Med 2008;67:1465-74. doi:10.1016/j.socscimed.2008.06.039

15 Parker MA, Robinson MHE, Scholefield JH, et al. Psychiatric morbidity and screening for colorectal cancer. J Med Screen 2002;9:7-10. doi:10.1136/ jms.9.1.7
16 Brasso K, Ladelund S, Frederiksen BL, et al. Psychological distress following fecal occult blood test in colorectal cancer screening--a population-based study. Scand J Gastroenterol 2010;45:1211-6. doi:10.310 9/00365521.2010.485355

17 Denters MJ, Deutekom M, Essink-Bot ML, et al. FIT false-positives in colorectal cancer screening experience psychological distress up to 6 weeks after colonoscopy. Support Care Cancer 2013;21:2809-15. doi:10.1007/ s00520-013-1867-7

18 Denters MJ, Deutekom M, Bossuyt PM, et al. Patient burden of colonoscopy after positive fecal immunochemical testing for colorectal cancer screening. Endoscopy 2013;45:342-9. doi:10.1055/s-0032-1326238

19 Kapidzic A, Korfage IJ, van Dam L, et al. Quality of life in participants of a CRC screening program. Br J Cancer 2012;107:1295-301. doi:10.1038/ bjc.2012.386

20 Mant D, Fitzpatrick R, Hogg A, et al. Experiences of patients with false positive results from colorectal cancer screening. Br J Gen Pract 1990;40:423-5-5.

21 Streiner DL, Norman GR. Health measurement scales, a practical guide to their development and use. 4th ed. Oxford University Press, 2008.

22 McCaffery KJ, Barratt AL. Assessing psychosocial/quality of life outcomes in screening: how do we do it better? J Epidemiol Community Health 2004;58:968-70. doi:10.1136/jech.2004.025114

23 DeFrank JT, Barclay C, Sheridan S, et al. The psychological harms of screening: the evidence we have versus the evidence we need. $J$ Gen Intern Med 2015;30:242-8. doi:10.1007/s11606-014-2996-5

24 Malmqvist J, Siersma V, Bang CW, et al. Consequences of screening in colorectal cancer (COS-CRC): development and dimensionality of a questionnaire. BMC Psychol 2021;9:7. doi:10.1186/s40359-020-00504-3

25 Vedsted P, Olesen F. A differentiated approach to referrals from general practice to support early cancer diagnosis - the Danish three-legged strategy. Br J Cancer 2015;112:S65-9. doi:10.1038/bjc.2015.44

26 Bie AKL, Brodersen J. Why do some participants in colorectal cancer screening choose not to undergo colonoscopy following a positive test result? A qualitative study. Scand J Prim Health Care 2018;36:262-71.

27 Brodersen J, Thorsen H. Consequences of screening in breast cancer (COS-BC): development of a questionnaire. Scand J Prim Health Care 2008;26:251-6.

28 Brodersen J, Thorsen H, Kreiner S. Validation of a condition-specific measure for women having an abnormal screening mammography. Value Health 2007;10:294-304.

29 Rasch G. Probabilistic models for some intelligence and attainment tests, 1960. Available: http://www.rasch.org/books.htm

30 Charlson ME, Pompei P, Ales KL, et al. A new method of classifying prognostic comorbidity in longitudinal studies: development and validation. J Chronic Dis 1987;40:373-83.

31 Statistics Denmark. Income, 2019. Available: https://www.dst.dk/da/ Statistik/emner/arbejde-indkomst-og-formue/indkomster/person-ogfamilieindkomster

32 Dufouil C, Brayne C, Clayton D. Analysis of longitudinal studies with death and drop-out: a case study. Stat Med 2004;23:2215-26. doi:10.1002/ sim. 1821

33 Benjamini Y, Hochberg Y. Controlling the false discovery rate: a practical and powerful approach to multiple testing. $J R$ Stat Soc Series B Stat Methodol 1995;57:289-300.

34 Osterø J, Siersma V, Brodersen J. Breast cancer screening implementation and reassurance. Eur J Public Health 2014;24:258-63.

35 Hestbech MS, Siersma V, Dirksen A, et al. Participation bias in a randomised trial of screening for lung cancer. Lung Cancer 2011;73:325-31.

36 The Danish colorectal cancer screening steering committee. The Danish colorectal cancer screening register: the annual report, 2018. Available: https://www.sundhed.dk/content/cms/45/61245_dts_årsrapport-2017_final. pdf

37 Toft EL, Kaae SE, Malmqvist J, et al. Psychosocial consequences of receiving false-positive colorectal cancer screening results: a qualitative study. Scand J Prim Health Care 2019;37:145-54.

38 Harris RP, Sheridan SL, Lewis CL, et al. The harms of screening: a proposed taxonomy and application to lung cancer screening. JAMA Intern Med 2014;174:281-5-5. doi:10.1001/jamainternmed.2013.12745 
39 Rasmussen JF, Siersma V, Pedersen JH, et al. Healthcare costs in the Danish randomised controlled lung cancer CT-screening trial: a registry study. Lung Cancer 2014;83:347-55.

40 Jensen MD, Siersma V, Rasmussen JF, et al. Direct and indirect healthcare costs of lung cancer CT screening in Denmark: a registry study. BMJ Open 2020;10:e031768.

41 Jodal HC, Helsingen LM, Anderson JC, et al. Colorectal cancer screening with faecal testing, sigmoidoscopy or colonoscopy: a systematic review and network meta-analysis. BMJ Open 2019;9:e032773. doi:10.1136/ bmjopen-2019-032773
42 King MT. A point of minimal important difference (MID): a critique of terminology and methods. Expert Rev Pharmacoecon Outcomes Res 2011;11:171-84. doi:10.1586/erp.11.9

43 Jaeschke R, Singer J, Guyatt GH. Measurement of health status. Control Clin Trials 1989;10:407-15. doi:10.1016/0197-2456(89)90005-6

44 Vermeer NCA, van der Valk MJM, Snijders HS, et al. Psychological distress and quality of life following positive fecal occult blood testing in colorectal cancer screening. Psychooncology 2020;29:1084-91. doi:10.1002/pon.5381

45 Korenstein D, Harris R, Elshaug AG, et al. To expand the evidence base about harms from tests and treatments. J Gen Intern Med 2021. doi:10.1007/s11606-021-06597-9. [Epub ahead of print: 21 Jan 2021]. 